\title{
Allocation Strategy for Wireless Communication Channel of Coal Mine Underground
}

\author{
Chao Zhang ${ }^{1,2}$ and Yi J. Zhang ${ }^{1}$ \\ ${ }^{1}$ China University of Mining and Technology, Xuzhou, China \\ ${ }^{2}$ Longkou Mineral Group Co. Ltd, Longkou, China \\ lunwenzzz@163.com
}

\begin{abstract}
This paper proposes an improved wireless communication channel reservation policy to address the impact on network performance cannot be analyzed under the original model with the primary and secondary users communication channel occupancy rates, and the problem that the secondary user blocking state is significantly higher than the primary user blocking probability under the original model. Simulation results show that improvement in network performance can be done by increasing the primary user communication channel occupy completion rate and the secondary user communication channel occupy completion rate. Also different users channel using request blocking probability can be reduced. It is more effective. That to improve the mixing network performance by improvement of secondary user communication channel occupy completion rate. The new improved model is important in ensuring the coal mine communication system (primary user) as the coal mine wireless communication system for emergency protection. Also the blocking probability of secondary users was reduced. The network access performance of the secondary user was enhanced and improved. The fairness among different users access to the hybrid network was ensured.
\end{abstract}

Keywords: the wireless communication, channel reserved

\section{Introduction}

Unlike traditional communication systems, wireless communication environment of coal mine underground is very harsh and the special [1-7]. The wireless signal propagation space is limited and irregular in the coal mine. Electromagnetic wave propagation in coal mine may not be in sight mostly. There is radio severe attenuation in the coal mine. And EMI noise interference also is serious in the roadway [8]. Although there are many available band theoretically, due to the tunnel effect and different electromagnetic waves propagating characteristics of underground coal mine roadway, the transmission band can be used for communication is not too wide and varied, so truly useful spectrum resources in coal mine underground are very limited.

Currently, the mine wireless communication systems are mainly through land mine VLF communication medium frequency induction communications, mining leakage communications systems, mine honeycomb cell system duplex wireless communication systems, PHS communication systems and power line carrier communication. Intense competition for different mixed wireless communication systems underground and limited radio resources, will lead to important communications systems (high priority) not working, such as coal mine underground wireless emergency communications and security systems. It will seriously jeopardize coal mine underground production safety $[9,10]$. Under such a 
network environment, how to effectively utilize radio resources, dynamic allocation of limited wireless resource, has brought great technical challenges for the wireless resource management. Radio resource management program based on policy is an effective method for the problem. This paper presents a service-related switching and management solutions for different types of businesses, with different switching strategies. The article gives a general approach for policy-based mechanism, and focus on next-generation wireless networks wireless resource management, policy-based network access control and switching mechanisms, and policy-based management quality of service mechanisms.

Channel allocation of wireless networks can be divided into fixed channel allocation (FCA), dynamic channel allocation (DCA) and hybrid channel assignment (HCA) categories [11-13]. To distinguish them, in this paper these are referred to channel allocation mechanism. Specific methods will be used on mechanism called the basis of channel allocation algorithm. Policy mechanism was first used in network security. With people's attention, strategies gradually applied in many fields. IETF also proposed a QoS policy-based management framework for policy control of isomorphic network service quality. Literature also presents a hybrid strategy for the UMTS network system. Documents will be extended to more general case.

In this paper, based on previous work, with the introduction of the policy mechanisms in channel allocation in wireless multimedia networks, based on the size of the network load, different channel allocation mechanism was used. In the wireless network, the reach strength of the same type operations in a different time of the day varies greatly. According to changes of business proportion, different channel allocation algorithms were used. In this paper, the channel allocation mechanism and channel assignment algorithm are collectively referred to channel allocation strategy. Combined with different channel allocation strategies together, a channel allocation scheme was formed. In other words, the core of policy-based channel allocation scheme is: according to the characteristics of the load status of the network and services, the channel allocation strategy was selected. And the wireless network available channel resources were flexible allocated and dynamically adjusted, so as to improve the utilization of radio resources and reduce the system blocking rate. Simulation results show that compared to using a single radio resource allocation scheme, using a policy mechanism to obtain better system performance.

\section{Original Wireless Communication Model}

In this scenario, the wireless network is a hybrid network, which uses a DAC (Dynamic Access Control) centralized wireless spectrum channels control method which contain two types of access user levels: PU (Primary Users) and SU (Secondary Users). Primary and secondary users take overlap way to share local radio spectrum resources (i.e., spectral overlap of the same time will produce communications interference), but the access priority of main user is higher than the second user.

According to scholars HUANG Z. said, in order to simplify the complexity of scene analysis, more assumptions will be introduced. Assuming that during hybrid network communication each time a user occupies only one communication channel. And channel resources usage request rate of primary and secondary users meet the Poisson process distribution, denoted $\lambda_{p}$ and $\lambda_{s}$. And the channel occupy time during communication meet Exponential distribution with mean which $\bar{T}=1 / \mu$.

Reserved channel strategy will be interpreted as a given local hybrid network of available radio spectrum resources channel number $\mathrm{N}$, which can be divided into ordinary channel $\mathrm{C}$ and reserved channel G. It satisfied that: 


$$
N=C+G
$$

When this hybrid network channel occupancy is less than or equal to $\mathrm{C}$, each user's new arrival channel resources requested are allowed access. While channel occupancy is greater than $\mathrm{C}$, secondary user channel resource using request will be blocked, but the main users are unaffected. When the channel occupancy is equal to $\mathrm{N}$ (i.e., unavailable channel resources), the channel resource requests of the primary and secondary users are blocked.

The theory scene can be used with one-dimensional Markov chain mathematical modeling analysis. If $\mathrm{E}(\mathrm{t})$ is defined as the state of hybrid network channels occupied number at time $\mathrm{t}$, $\{\mathrm{E}(\mathrm{t}), \mathrm{t} \geq 0\}$ Represents a continuous time one-dimensional Markov chain which has a total of $\mathrm{N}+1$ finite number of states from $\mathrm{N}$ to 0 . We put $\mathrm{P}(\mathrm{i})$ referred as the steady state probability of $i$, then $P(j)$ satisfies:

When this hybrid network channel occupancy is less than or equal to $\mathrm{C}$, each user's new arrival channel resources requested are allowed access. While channel occupancy is greater than $\mathrm{C}$, secondary user channel resource using request will be blocked, but the main users are unaffected. When the channel occupancy is equal to $\mathrm{N}$ (i.e., unavailable channel resources), the channel resource requests of the primary and secondary users are blocked.

The theory scene can be used with one-dimensional Markov chain mathematical modeling analysis. If $\mathrm{E}(\mathrm{t})$ is defined as the state of hybrid network channels occupied number at time $\mathrm{t}$, $\{\mathrm{E}(\mathrm{t}), \mathrm{t} \geq 0\}$ Represents a continuous time one-dimensional Markov chain which has a total of $\mathrm{N}+1$ finite number of states from $\mathrm{N}$ to 0 . We put $\mathrm{P}(\mathrm{i})$ referred as the steady state probability of $i$, then $P(j)$ satisfies:

$$
P(i)=\lim _{t \rightarrow \infty} P\{E(t), t=i\}
$$

The state transition diagram was shown in Figure 1.

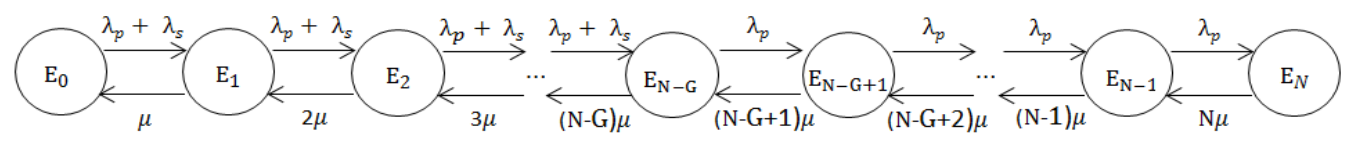

Figure 1. Guard Channel State Transition Diagram

The recursive form of $\mathrm{P}(\mathrm{i})$ was shown as:

$$
P(i)=\left\{\begin{array}{c}
\frac{\lambda_{p}+\lambda_{s}}{i_{\mu}} P(i-1), i=1,2,3, \ldots, N-G \\
\frac{\lambda_{p}}{i_{\mu}} P(i-1) i=N-G+1, \ldots N
\end{array}\right.
$$

And satisfies:

$$
\sum_{i=0}^{\infty} P(i)=1
$$

According to the initial value $\mathrm{P}(0)$, the iterative $\mathrm{P}(\mathrm{i})$ can be rewritten as:

$$
P(i)=\left\{\begin{array}{c}
\frac{\left(\lambda_{p}+\lambda_{s}\right) i}{i_{\mu} ! \mu i} P(0), i=1,2,3, \ldots, N-G \\
\frac{\left(\lambda_{p}+\lambda_{s}\right) N-G \lambda p(i-N+G)}{i_{\mu} ! \mu i} P(0) i=N-G+1, \ldots N
\end{array}\right.
$$




$$
P(0)=\left\lceil\sum_{j=0}^{N-G} \frac{(\lambda p+\lambda s) j}{j ! \mu j}+\sum_{j=N-G+1}^{N} \frac{\left(\lambda_{p}+\lambda_{s}\right)^{N-G} \lambda_{p}{ }^{(i-N+G)}}{j ! \mu j}\right\rceil^{-1}
$$

According to the state transition diagram, it can be obtained that the blocking probability $\mathrm{BP}(\mathrm{G}, \mathrm{N})$ of the primary user is the state probability of hybrid network with the channel occupancy number $\mathrm{N}$, which was given as:

$$
\begin{aligned}
B P(G, N) & =P(N) \\
& =\left\lfloor\frac{a^{N-G} b^{G}}{N !}\right\rfloor /\left\lfloor\sum_{i=0}^{N-G-1} \frac{a i}{i !}+\sum_{i=N-G}^{N} \frac{a^{N-G}}{i !} B^{i-N+G}\right\rfloor
\end{aligned}
$$

The secondary user blocking probability $\mathrm{BS}(\mathrm{G}, \mathrm{N})$ is the sum of the state probability of which hybrid network channel occupancy is equal to or greater than $\mathrm{C}(\mathrm{N}-\mathrm{G})$, which was given as:

$$
\begin{aligned}
B S(G, N)= & \sum_{i=N-C}^{N} P(i) \\
& \frac{a^{N-G}}{(N-G) !}+a^{N-G} \sum_{i=1}^{G} \frac{b i}{(N-G+i) !} \\
& \sum_{i=0}^{N-G} \frac{a i}{i !}+a^{N-G} \sum_{i=1}^{G} \frac{b i}{(N-G+i) !} \\
& a=\frac{\lambda p+\lambda s}{\mu}, b=\frac{\lambda p}{\mu}
\end{aligned}
$$

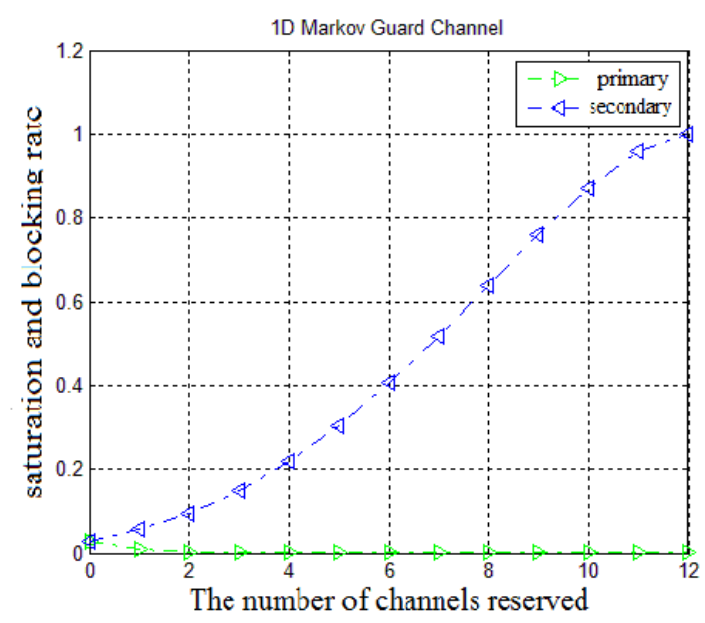

\section{Figure 2. Diagram of Simulation Results}

This article uses the following parameter assignment. Local hybrid network available radio spectrum resources channel number $\mathrm{N}$ is 12 . The channel resource request rate of primary users $\lambda p$ is 2 . The channel resource request rate of secondary user's $\lambda s$ is 5 . Communication channel occupies a frequency $\mu$ with value of 1 . Matlab simulation results were shown in Figure 2.

It can be seen from assignment parameters of this section, the mathematical model does not distinguish the communication channel occupancy rate $\mu$ of primary and secondary users, which does not fit the actual communication applications. While it is not conducive for the analysis of affect on the network performance from the occupancy completion rate of the 
communication channel different primary and secondary users. At the same time, it can be seen from Figure 2, when the number of channels reserved was up to 7, the channel saturation and blocking probability of secondary user has exceeded 50\%, which means that there will be more than half of the secondary user channel call request was rejected. It is not conducive to the network access fairness principle. To reduce the blocking probability of higher secondary users, the improved channel access reservation control strategy would be introduced.

\section{Improved Wireless Communication Model}

The channel allocation mechanism and channel allocation algorithm were used in this article. The channel allocation mechanisms are dynamic channel allocation and dynamic channel allocation. The following describes the channel allocation algorithm used here. The first algorithm used in this paper is based on the pre-emptive priority combined with guaranteed channel, called PGC.

This paper firstly focuses on channel allocation algorithm in the case of dynamic channel allocation. In the case of dynamic channel allocation, taking into account the presence of cochannel interference, even if there is an idle channel, the user's access request may be denied. Take $f(i)$ and $g(j)$ ( $i$ and $j$ represent the number of primary users and secondary users) to represent the rejected probability of primary and secondary users access request. Blocking rate formula can be introduced as:

First, make the definition of $\mu \mathrm{PH}$ and $\mu \mathrm{SH}$ :

$$
\left\{\begin{array}{l}
1 / \mu_{P H}=1 /\left(\mu_{V}+\mu_{V W}\right) \\
1 / \mu_{S H}=1 /\left(\mu_{D}+\mu_{D W}\right)
\end{array}\right.
$$

For PGC, assuming the number of channels reserved for handover is $\mathrm{CH}, \mathrm{f}(\mathrm{i})$ and $\mathrm{g}(\mathrm{j})$ can be expressed as:

$$
\begin{aligned}
& f(i)=\sum_{j=0}^{7 C+Q_{s}-i} b(i+j) P_{1}(j \mid i) \\
& g(j)=\sum_{i=0}^{7 C+Q_{p}-j} b(i+j) P_{1}(j \mid i)
\end{aligned}
$$

Where $b(i+j)$ can be defined as:

$$
b(i+j)=\left\{\begin{array}{l}
{\left[1-\left(1-\omega_{1}\right) g\right]^{\left.7\left(C-C_{H}\right)-(i+j) / R\right]},} \\
0 \leq i+j \leq 7\left(C-C_{H}\right) ; \\
{\left[1-\left(1-\omega_{2}\right) g\right]^{7 C-(i+j) / R]},} \\
0 \leq i \leq 7 C, \\
7\left(C-C_{H}\right)<i+j \leq 7 C+Q_{S} ; \\
{\left[1-\left(1-\omega_{2}\right) g\right]^{7 C-(i+j) / R]},} \\
0 \leq j \leq 7 Q_{S}
\end{array}\right.
$$

Where,

$$
\begin{aligned}
\omega_{1} & =\frac{\left(1-P_{P N}\right) D_{1}+\left(1-P_{P H}\right) D_{2}+\left(1-P_{S N}\right) D_{3}}{7\left(C-C_{H}\right)(g+1)} \\
& =+\frac{\left(1-P_{S H}\right) D_{4}}{7\left(C-C_{H}\right)(g+1)}
\end{aligned}
$$


International Journal of Future Generation Communication and Networking

$$
\begin{gathered}
\omega_{2}=\frac{P_{P N}\left(1-P_{P N}\right) D_{1}+P_{P H}\left(1-P_{P H}\right) D_{2}+P_{S N}\left(1-P_{S N}\right) D_{3}+}{7 C_{H}(g+1)} \\
=\frac{+P_{S H}\left(1-P_{S H}\right) D_{4}}{7 C_{H}(g+1)} \\
\omega_{3}=\frac{P_{P H}\left(1-P_{P H}\right) D_{2}+P_{S N}\left(1-P_{S N}\right) D_{3}}{7 C_{H}(g+1)} \\
=\frac{+P_{S H}\left(1-P_{S H}\right) D_{4}}{7 C_{H}(g+1)}
\end{gathered}
$$

And:

$$
\begin{gathered}
D_{1}=\frac{\lambda_{P N}}{\mu_{P}+\mu_{P W}}\left(1+\frac{g}{R}\right) \quad D_{2}=\frac{\lambda_{P H}}{\mu_{P}+\mu_{P W}}\left(1+\frac{g}{R}\right) \\
D_{3}=\frac{\lambda_{S N}}{\mu_{S}+\mu_{S W}}\left(1+\frac{g}{R}\right) \quad D_{4}=\frac{\lambda_{S N}}{\mu_{S}+\mu_{S W}}\left(1+\frac{g}{R}\right)
\end{gathered}
$$

Make $[(\mathrm{i}+\mathrm{j}) / \mathrm{R}]$ to represent the lower limit of $(\mathrm{i}+\mathrm{j}) / \mathrm{R}, \mathrm{P} 1(\mathrm{j} \mid \mathrm{i})$ was defined as:

$$
P_{1}(j \mid i)=\left\{\begin{array}{c}
\frac{\lambda_{S N j}+\lambda_{S H j}}{j \mu_{S H}} P_{1}(j-1 \mid i) 1 \leq j \leq 7 C-i \\
\frac{\lambda_{S H j}}{j \mu_{S W}} P_{1}(j-1 \mid i) 7 C+1-i \leq j \leq 7 C+Q_{S}-i
\end{array}\right.
$$

Where,

$$
\lambda_{S N j}=7 \lambda_{S N}[1-g(j)], \quad \lambda_{S H j}=\left(7 \lambda_{S H}+\lambda_{S P}\right)[1-g(j)]
$$

Make $\mu \mathrm{PQ}$ represents the primary user average waiting time in the queue, and P2(i) was defined as:

$$
P_{2}(i)=\left\{\begin{array}{l}
\frac{\lambda_{P N i}+\lambda_{P H i}}{j \mu_{P H}} P_{2}(i-1) \quad 1 \leq i \leq 7\left(C-C_{H}\right) \\
\frac{\lambda_{P H i}}{i \mu_{P H}} P_{2}(i-1) \quad 7\left(C-C_{H}\right)+1 \leq i \leq 7 C \\
\frac{\lambda_{P H i}}{i \mu_{P Q}} P_{2}(i-1) \quad 7 C+1 \leq i \leq 7 C+Q_{P}
\end{array}\right.
$$

Where:

$$
\lambda_{P N i}=7 \lambda_{P N}[1-f(i)], \lambda_{P H i}=7 \lambda_{P H}[1-f(i)]
$$

Considering the primary user has preemptive priority, the new call and handover blocking rate of the primary users, and new call and handover blocking rate of secondary users can be given respectively by the following formula:

$$
\begin{aligned}
P_{P N}= & \sum_{i=0}^{7\left(C-C_{H}\right)}\left[P_{2}(i) f(i)\right]+\sum_{i=7\left(C-C_{H}\right)+1}^{7 C+Q_{P}} P_{2}(i) \\
P_{P H} & =\sum_{i=0}^{7 C+Q_{P}-1}\left[P_{2}(i) f(i)\right]+P_{2}\left(7 C+Q_{P}\right) \\
& +\sum_{i=7 C+1}^{7 C+Q_{P}}\left[\frac{\mu_{P Q}(i-7 C) P_{2}(i)}{\lambda_{P H}(1-f(i))}\right]
\end{aligned}
$$




$$
\begin{aligned}
P_{S N}= & \sum_{i=0}^{7 C} \sum_{j=0}^{7 C-i}\left[P_{1}(j \mid i) P_{2}(i) g(j)\right]+\sum_{i=0}^{7 C} \sum_{j=7 C-i+1}^{7 C+Q_{S}-i}\left[P_{1}(j \mid i) P_{2}(i)\right] \\
& +\sum_{i=7 C+1}^{7 C+Q_{P} Q_{S}}\left[P_{j=1}(j \mid i) P_{2}(i)\right] \\
P_{S H}= & \left(1+\frac{\lambda_{S P}}{7 \lambda_{S H}+\lambda_{S P}}\right)\left\{\sum_{i=0}^{7 C} \sum_{j=0}^{7 C+Q_{S}-i-1}\left[P_{1}(j \mid i) P_{2}(i) g(j)\right]\right. \\
& \left.+\sum_{i=7 C+1}^{7 C+Q_{P} Q_{S}}\left[P_{1}(j \mid i) P_{2}(i) g(j)\right]\right\} \\
& +\left(1+\frac{\lambda_{S P}}{7 \lambda_{S H}+\lambda_{S P}}\right) \sum_{i=0}^{7 C}\left[P_{1}\left(7 C+Q_{S}-i \mid i\right) P_{2}(i)\right]
\end{aligned}
$$

Average queue length of primary user E[LP], average queue length of secondary user $\mathrm{E}[\mathrm{LD}]$ and $\lambda \mathrm{SP}$ can be expressed as:

$$
\begin{gathered}
E\left[L_{P}\right]=\sum_{i=7 C+1}^{7 C+Q_{P}}\left[(i-7 C) P_{2}(i)\right] \\
E\left[L_{D}\right]=\sum_{i=0}^{7 C} \sum_{j=7 C+1-i}^{7 C+Q_{D}-i}\left[\left(7 C+Q_{D}-i-j\right) P_{2}(i) P_{1}(j \mid i)\right] \\
+\sum_{i=7 C+1} \sum_{j=1}\left[j P_{2}(i) P_{1}(j \mid i)\right] \\
\lambda_{D P}=7 \lambda_{V N} \sum_{i=0}^{7\left(C-C_{H}\right)-17 C+Q_{V}-i} \sum_{j=1}\left[P_{2}(i) P_{1}(j \mid i)\right] \\
+3 \lambda_{V H} \sum_{i=0}^{7 C-17 C+Q_{V}-i} \sum_{j=1}\left[P_{2}(i) P_{1}(j \mid i)\right]
\end{gathered}
$$

Assume that each user has 12 channels. Primary and secondary users are assumed queue length is 7. In the PGC program for real-time channel reserved for handover request number is 14. Set other parameters as: $\mathrm{TVW}=\mathrm{TDW}=60 \mathrm{~s}, \mathrm{TV}=\mathrm{TD}=120 \mathrm{~s}, \mathrm{TVQ}=5 \mathrm{~s}$. This paper considers the scenario that the arrival intensity of the main user is not close to intensity of secondary user (set $\lambda \mathrm{VN}: \lambda \mathrm{DN}=3: 1$ ). Figure shows that blocking rates of the primary and secondary users when the arrival rates are relatively different. The arrival ratio here of the primary and secondary users is 3:1. Figure 3(a) shows the primary user blocking rate curve; while the Figure 3(b) gives the secondary user blocking rate curve. For convenience, the figure used different symbols to represent different strategies, such as FCA (Fixed Channel Allocation), DCA (Dynamic Channel Allocation). PN and PH represent the new call and handover user of the primary, while SN and SH represent new call and handover of the secondary user. Final selection strategies expressed by Policy. 


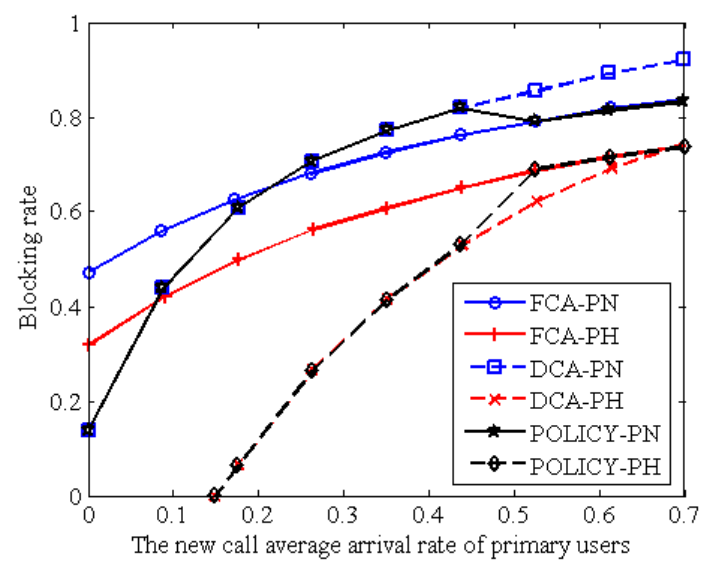

(a) The Blocking Probabilities of Primary User

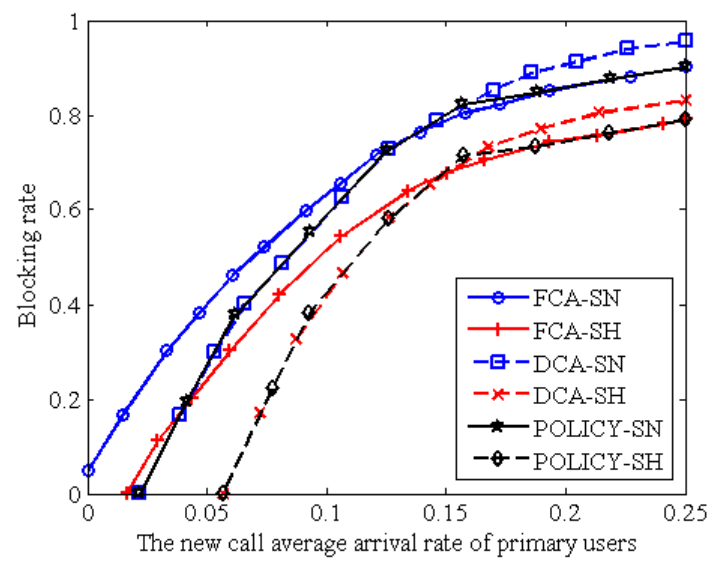

(b) The Blocking Probabilities of Secondary User

Figure 3. Simulation Results of the Blocking Probabilities

In the case of to ensure a proportion of the secondary user can access the network, the blocking probability of the primary user can also be controlled within an acceptable range. For the lower reaching intensity of the primary and secondary users, dynamic channel allocation mechanism was chosen; while the reaching intensity of the primary and secondary users is relatively high, a fixed channel allocation mechanism was chosen. In doing so, the blocking probability is further reduced.

\section{Experimental Results}

In order to facilitate comparative analysis, local hybrid network available radio spectrum resources channel number $\mathrm{N}$ was 12 . The channel resource request rate of primary user's $\lambda \mathrm{p}$ is 2. The channel resource request rate of secondary user's $\lambda s$ is 5 .

Firstly, the effect of the primary user communications channels occupied completion rate $\mu \mathrm{p}$ and secondary user communication channel occupancy completion rate $\mu$ s on the primary user network performance was analyzed. The simulation results of primary user saturation and blocking rate in the formation of different primary user's communication channel occupancy completion rate $\mu$ p were shown in Figure 4. 


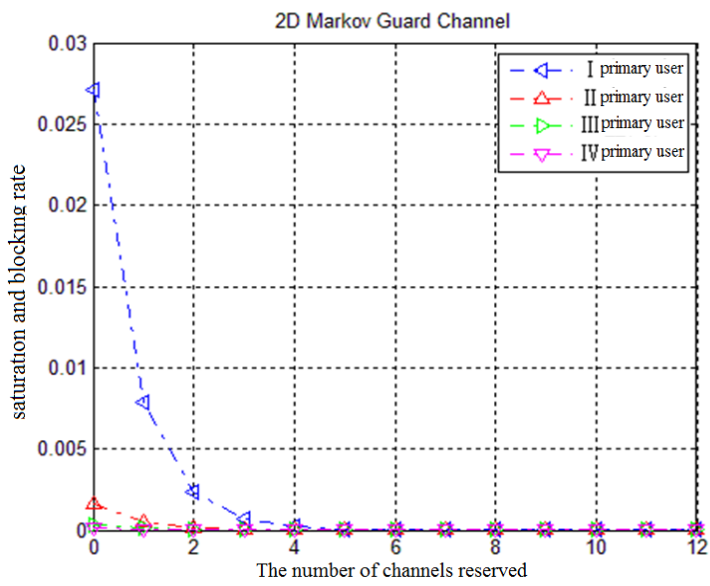

Figure 4. Different $\mu p$ to Primary User Performance

In the Figure 4 above, the blue dotted line represents $I$ : the primary user saturation and blocking rate under the primary user communications channels occupied completion rate $\mu \mathrm{p}=$ 1; red dotted line represents II : the primary user saturation and blocking rate under the primary user communications channels occupied completion rate $\mu \mathrm{p}=2$; green dotted line represents III: the primary user saturation and blocking rate under the primary user communications channels occupied completion rate $\mu p=3$; pink dotted line represents IV: the primary user saturation and blocking rate under the primary user communications channels occupied completion rate $\mu p=4$. It can be seen from the comparison chart, the saturation and blocking rate of the primary user becomes lower with the increasing of primary user communications channels occupied completion rate $\mu$ p.

The simulation results of primary user saturation and blocking rate in the formation of different primary user's communication channel occupancy completion rate $\mu$ s were shown in Figure 4.

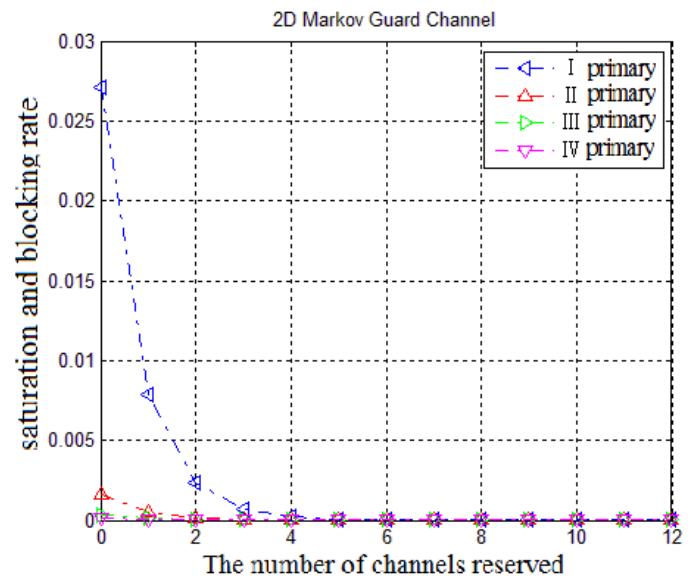

Figure 5. Different $\mu$ s to Primary User Performance

In the Figure 5 above, the blue dotted line represents $I$ : the primary user saturation and blocking rate under the secondary user communications channels occupied completion rate $\mu \mathrm{s}$ $=1$; red dotted line represents II : the primary user saturation and blocking rate under the 
secondary user communications channels occupied completion rate $\mu \mathrm{s}=2$; green dotted line represents III: the primary user saturation and blocking rate under the secondary user communications channels occupied completion rate $\mu \mathrm{s}=3$; pink dotted line represents IV: the primary user saturation and blocking rate under the secondary user communications channels occupied completion rate $\mu \mathrm{s}=4$. It can be seen from the comparison chart, the saturation and blocking rate of the primary user becomes lower with the increasing of secondary user communications channels occupied completion rate $\mu$ s.

It should be noted that it can be seen from the comparison of Figures 4 and 5, the effect on the network performance by the secondary user communications channels occupied completion rate $\mu$ s is more obvious than it by the primary user communications channels occupied completion rate $\mu$ p

The following simulation will show the comparative analysis of the improved wireless channel reservation policy and the original model. To facilitate comparative analysis, parameters assignment was as the same before. Simulation results of comparison between the improved wireless channel reservation policy and the original model was shown in Figure 6 as follows.

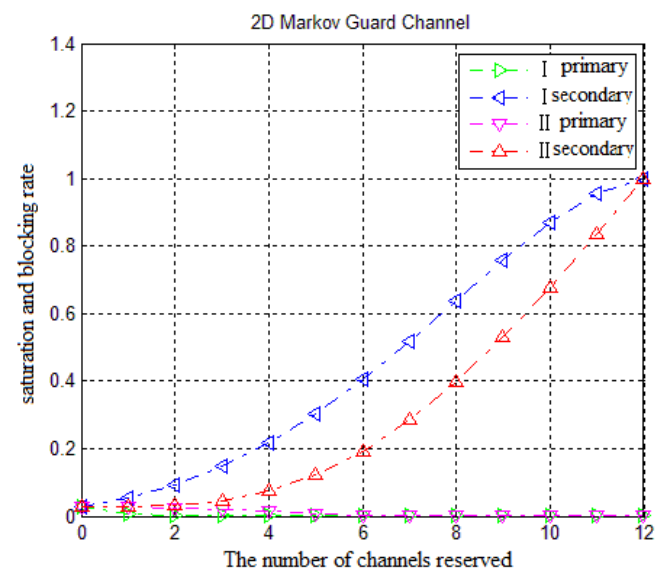

Figure 6. Comparison of Simulation Results

In comparison chart, the green dotted line represents the primary user saturation and blocking probability of the original model; pink dotted line represents the primary user saturation and blocking probability of improved model; blue dotted line represents the secondary user saturation and blocking probability of the original model; the red dotted line represents the secondary user saturation and blocking probability of improved model. It is still reserved channel value equal to 7 . The values of secondary user saturation and blocking probability in the new state model have been reduced to 0.3 . There are no abnormal changes with saturation and blocking probability of the primary user. As it can be seen in the new improved model, of the situation to ensure that the main user communication was substantially unaffected, the blocking probability of secondary users was successfully reduced. The network access performance of the secondary user was improved.

\section{Conclusions}

This paper proposes an improved wireless communication channel reservation policy to address the impact on network performance cannot be analyzed under the original model with the primary and secondary users communication channel occupancy rates, and the problem 
that the secondary user blocking state is significantly higher than the primary user blocking probability under the original model. Simulation results show that improvement in network performance can be done by increasing the primary user communication channel occupy completion rate and the secondary user communication channel occupy completion rate. Also different users channel using request blocking probability can be reduced. It is more effective. That to improve the mixing network performance by improvement of secondary user communication channel occupy completion rate. The new improved model is important in ensuring the coal mine communication system (primary user) as the coal mine wireless communication system for emergency protection. Also the blocking probability of secondary users was reduced. The network access performance of the secondary user was enhanced and improved. The fairness among different users access to the hybrid network was ensured.

\section{References}

[1] M. Ghaderi and R. Boutaba, "Call admission control for voice/data integration in broadband wireless networks”, IEEE Transactions on Mobile Computing, vol. 5, no. 3, (2006) March, pp. 193-207.

[2] Y. Li, S. McLaughlin, D. G. M. Cruickshank and X. Wei, "Towards multi-mode terminals", IEEE Vehicular Magazine, vol. 1, no. 4, (2006) December, pp. 17-24.

[3] H. Bing, C. He and L. Jiang, "Performance analysis of vertical handover strategy for heterogeneous overlay network", Chinese Journal of Electronics, vol. 13, no. 4, (2004) October, pp. 623-628.

[4] W. Zhao, R. Tafazolli and B. G. Evans, "Internetwork handover performance analysis in a GSM-Satellite integrated mobile communication system", IEEE Journal on Selected Areas in Communications, vol. 8, no. 15, (1997) October, pp. 1657-1671.

[5] X. Duan, Z. Niu and J. Zheng, "Optimizing radio resource allocation in multimedia DS-CDMA systems based on utility functions", IEICE Transaction on Communications, vol. E87-B, no. 8, (2004) August, pp. 2233-2242.

[6] X. Duan, Z. Niu and J. Zheng, "Capacity analysis of uplink and downlink in multimedia DS-CDMA systems based on constraint models", IEEE International Conference on Communications, vol. 1, (2003) May, pp. 402-406, Alaska, USA.

[7] E. N. Onwuka and Z.-S. Niu, "Service dependent handoff management for efficient mobile IP communication", IEEE International Conference on Networking, Sensing and Control, (2004) March, pp. 21-23, Tai Pei, China.

[8] C. Hsiung, "Inter-harmonic identification using group- Harmonic weighting approach based on the FFT," IEEE Trans on Power Electronics, vol. 23, no. 3, (2008) May, pp. 1309- 1319.

[9] R. Yavatkar, D. Pendarakis and R. Guerin, “A framework for policy based admission control”, RFC 2753, (2000) January.

[10] Y. Snir, Y. Ramberg, J. Strassner and R. Cohen, "Policy framework QoS information model", (2001) November.

[11] W. Zhuang, Y.-S. Gan, Q. Gao, et al., "Multi-domain policy architecture for IP multimedia subsystem in UMTS", Conference on Network Control and Engineering for QoS, Security and Mobility, (2002), pp. 2738.

[12] W. Yue, "Analytical methods to calculate the performance of a cellular mobile radio communication system hybrid channel assignment”, IEEE Transactions on Vehicular Technology, vol. 40, no. 2, (1991) May, pp. 453-460.

[13] Z. H. Zheng and W. H. Lam, "Analytical methods to calculate performance of handoff prioritization in dynamic channel assignment," Electronics Letters, July 2001, vol. 37, no. 15, (2001) July, pp. 978-979.

[14] Z. H. Zheng and W. H. Lam, "Performance analysis of dynamic channel assignment with queuing and guard channel combined scheme for handoff prioritization," Electronics Letters, vol. 38, no. 25, (2002) December, pp. 1728-1729. 


\section{Authors}

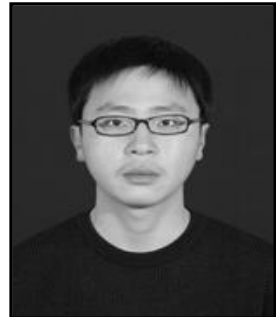

Chao Zhang, he was born in Zou Cheng, Shangdong, China in 1987. He received his B.S. and M.S. from the Shangdong University of Science and Technology, China, in 2008 and 2011, respectively. Since 2011, he has been working towards his Ph.D. in the Department of Information and Electrical Engineering, China University of Mining and Technology. His current research interests include intelligent control, power quality, large capacity SAPF under complex network environment.

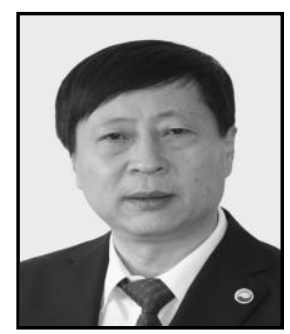

Yi J. Zhang, he was born in Tai An, Shangdong, China in 1961. He received the B.E. degree from the Shangdong University of Science and Technology, China. Now he is working in Longkou Mineral Group Co. Ltd. He is mainly engaged in coal power system security and management. 